B. SHAN $1,2, \infty$

A. CAVALIERI ${ }^{1}$

Z. CHANG $^{1,2}$

\title{
Tunable high harmonic generation with an optical parametric amplifier
}

\author{
${ }^{1}$ Center for Ultrafast Optical Science, University of Michigan, 1006 IST Building, 2200 Bonisteel Blvd., \\ Ann Arbor, MI 48109, USA \\ 2 Dept. of Physics, Kansas State University, 116 Cardwell Hall, Manhattan, KS 66502, USA
}

\begin{abstract}
Received: 16 September 2001/
Revised version: 11 November 2001

Published online: 27 June 2002 • (C) Springer-Verlag 2002

ABSTRACT A high-intensity $10^{14} \mathrm{~W} / \mathrm{cm}^{2}$ optical parametric amplifier (OPA) was developed to generate tunable infrared pulses for high harmonic generation (HHG). The HHG experiments with xenon and argon gases using the OPA output were performed with a $\mathrm{kHz}$ gas valve. The tunable nature of the OPA led to tunable $\mathrm{HHG}$, resulting in a source of fully tunable coherent XUV radiation from $0.22 \mu \mathrm{m}$ to the HHG cutoff. Compared with the conventional $800 \mathrm{~nm}$ (Ti:sapphire) driving field, increasing the driving wavelength by an OPA also significantly extended the cutoff photon energy of the HHG.
\end{abstract}

PACS 32.80.Rm; 42.65.Ky

1

\section{Introduction}

High harmonic generation (HHG) can produce coherent XUV/X-ray radiation that has many important potential applications, such as ultrafast femtosecond spectroscopy and microscopy $[1,2]$. HHG is a non-perturbative process that occurs only when the incident excitation field is comparable to the Coulomb field within an atom [3,4]. Therefore, production of $\mathrm{XUV} / \mathrm{X}$-rays by $\mathrm{HHG}$ in noble gases requires laser intensities above $10^{13} \mathrm{~W} / \mathrm{cm}^{2}$. The most commonly used lasers to provide such intensities are Ti:sapphire and Nd:YAG/glass lasers, with carrier wavelengths of 0.8 and $1.06 \mu \mathrm{m}$, respectively.

Since HHG produces only odd harmonics of the driving laser, there are inherent limitations on the XUV radiation produced by fixed-wavelength pump lasers. The XUV radiation spectrum produced is discrete. This limits the potential applications of a HHG X-ray source. The output spectrum of the HHG experiment can be tuned to cover the gap between adjacent orders by using a tunable excitation field. Harmonic generation in the 1.2 to $1.55 \mu \mathrm{m}$ range was achieved using an OPA operating at $10^{12} \mathrm{~W} / \mathrm{cm}^{2}$ [5]. But at this low intensity the highest harmonic observed was the 9 th harmonic

Fax:+1-734/763-4876, E-mail: bings@phys.ksu.edu
( $\sim 8 \mathrm{eV}$ photon). For the first time, we demonstrated HHG in the XUV/soft X-ray regime using a high-intensity OPA.

The high-intensity OPA also allowed us to study HHG in a pump wavelength regime comparably longer than $1.06 \mu \mathrm{m}$ (Nd:YAG). The semi-classical model $[6,7]$ of $\mathrm{HHG}$ predicts that the cutoff photon energy is $h v_{\text {cutoff }}=I_{\mathrm{p}}+3.17 U_{\mathrm{p}}$, where $I_{\mathrm{p}}$ is the ionization energy and $U_{\mathrm{p}}$ is the ponderomotive energy. Since the ponderomotive energy is proportional to the square of the wavelength of the incident field, we expect significantly higher cutoff photon energies for pump lasers of longer wavelength.

\section{$2 \quad$ Experiment setup \\ 2.1 System layout}

Figure 1 shows the experimental setup. A Ti:sapphire laser system that produced $1.2 \mathrm{~mJ}, 21 \mathrm{fs}$ pulses at sub$\mathrm{kHz}$ repetition rate [8] is used to pump the OPA. The OPA generates tunable 1.1 to $1.6 \mu \mathrm{m}$ IR laser pulses, and was focused by an $88.3 \mathrm{~mm}$ lens onto the pulsed gas jet formed by a gas valve synchronized with the laser signal. The focal spot size is $\sim 20 \mu \mathrm{m}$ FWHM. The gas density from the pulsed jet is $\sim 1 \times 10^{18}$ atoms $/ \mathrm{cm}^{3}$ in a $200 \mu \mathrm{m}$ interaction region. The high harmonic signal, after passing a pinhole and a slit, was imaged by a focusing mirror at grazing incidence onto a chevron MCP imaging detector that has good sensitivity to wavelengths below $140 \mathrm{~nm}$. A $2000 \mathrm{l} / \mathrm{mm}$ transmission grating is employed to disperse the spectrum. Finally, the Xray spectrum on the phosphor screen was recorded by a 16 bit cooled CCD camera.

In the system, the MCP detector required a high vacuum (better than $10^{-6}$ ) to apply high voltages across the electrodes. However, HHG needs a rather high gas density in the laser-gas interaction region. Therefore, the spectrometer and the gas nozzle were installed in two separate vacuum chambers. The two chambers were separated by a $0.5 \mathrm{~mm}$ pinhole that allowed the HHG signal to pass through to the detector chamber with relatively little gas leakage. We also employed a $\mathrm{kHz}$ gas valve that produced a smaller gas load on the vacuum system. This differential pumping scheme allowed both a good vacuum in the detector chamber and a high enough transient local density in the interaction region. 


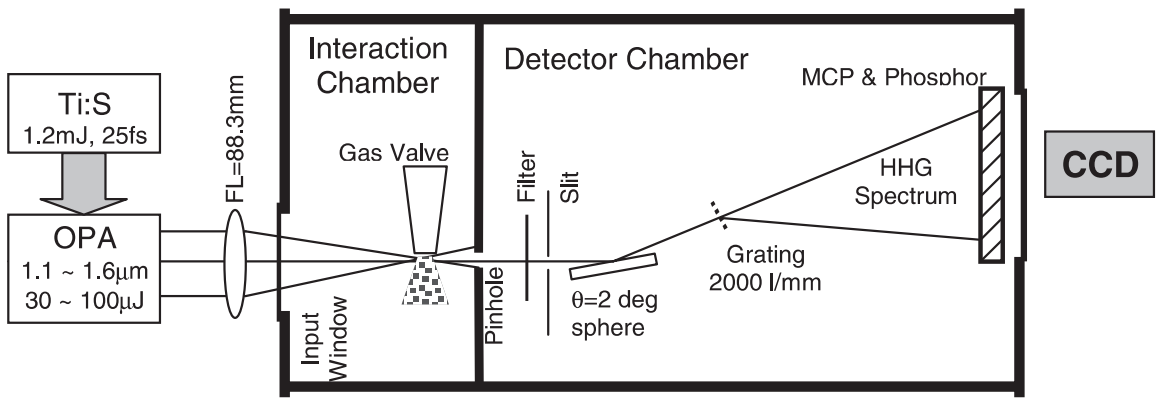

FIGURE 1 Experimental setup

\section{2}

\section{$\mathrm{kHz}$ gas valve}

The working medium in HHG is usually provided by a static gas cell [9], a hollow fiber [10], or a high repetition gas valve [11]. The pulsed gas valve yielded a simple and efficient way to produce a high-density gas in the focal volume. The pulsed gas valve we used is a solenoid valve that can operate at high repetition rate (up to $1.2 \mathrm{kHz}$ ). It is commercially available and is much cheaper than a piezoelectric valve [12].

The performance of the valve was measured by observing the ionization fluorescence [13] when a laser was focused into the gas produced by the valve with a suitable intensity. Figure 2 shows the measured temporal and spatial profiles of the
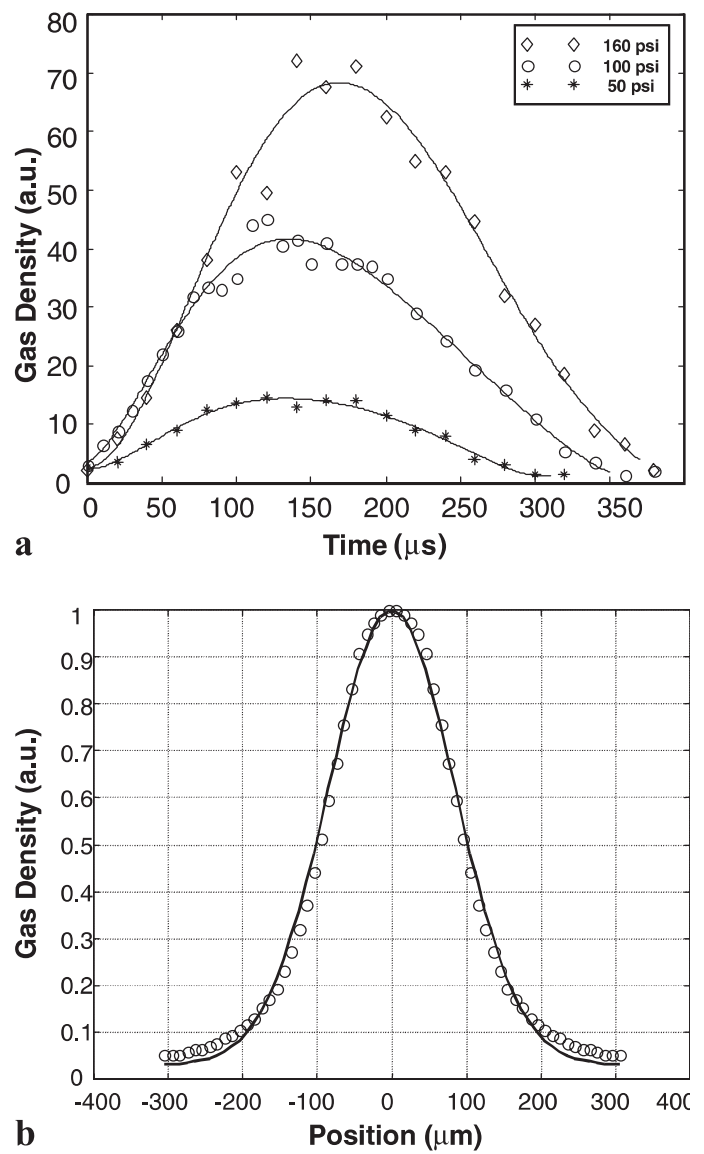

FIGURE 2 Characteristics of the $\mathrm{kHz}$ gas valve. a Temporal profile of the gas pulse from the valve. The signal was measured at three different backing pressures with argon gas. b Measured spatial distribution. The position is the horizontal offset from the nozzle center. The Gaussian fitting (line) gives a distribution width of $\sim 180 \mu \mathrm{m}$ gas pulse. As shown in the figure, the duration of the gas pulse is $\sim 200 \mu \mathrm{s}$, and the time at which peak density occurs changes slightly with the backing pressure. The gas density is proportional to the backing pressure. The spatial profile at the time of peak density is nearly Gaussian with a FWHM of $\sim 180 \mu \mathrm{m}$.

\section{$3 \quad$ High-intensity OPA}

We modified a commercially available OPA to accept shorter and more intense input pulses from our Ti:sapphire laser system. The output signal of the OPA was tunable from 1.1 to $1.6 \mu \mathrm{m}$. The pulse energy at the output of the OPA (signal+idler) was $400 \mu \mathrm{J}$ at a signal wavelength of $1.51 \mu \mathrm{m}$. After the signal propagated a relatively long distance to the HHG system, the pulse energy of the signal dropped to $150 \mu \mathrm{J}$ due to the divergence of higher-order spatial modes and absorption/scattering in the path.

To characterize the $1.51 \mu \mathrm{m}$ output from the OPA we measured the spectrum, near field profile and far field pattern of the beam. The spectrum was measured with a monochro-
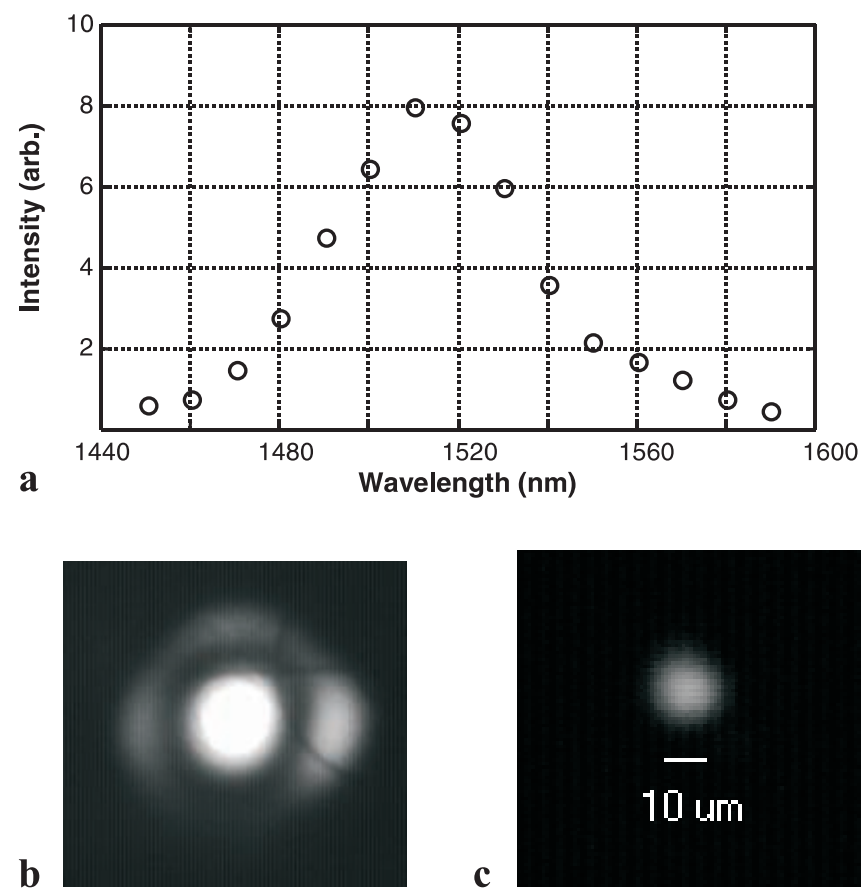

FIGURE 3 Output characteristics of the OPA with $1.51 \mu \mathrm{m}$ output. a Spectrum. b Near field profile. The output pulse energy is $\sim 400 \mu \mathrm{J}$. c Far field pattern focused by an $88.3 \mathrm{~mm}$ lens. The intensity at the focal point is $\sim 3 \times 10^{14} \mathrm{~W} / \mathrm{cm}^{2}$ 
mator and an InGaAs photodiode that has a flat response up to $1660 \mathrm{~nm}$. The FWHM of the distribution is $\sim 50 \mathrm{~nm}$, as shown in Fig. 3a. The near field profile is shown in Fig. 3b. To observe the far field we focused the beam with an $88.3 \mathrm{~mm}$ lens and measured the focal point profile with a commercial $\mathrm{Si}$ $\mathrm{CCD}$ and a $10 \times$ objective lens. Figure $3 \mathrm{c}$ is the recorded profile. The FWHM of the measured image is $12 \mu \mathrm{m}$. At high intensity, the Si CCD responded to the $1.51 \mu \mathrm{m}$ signal through two-photon absorption, therefore the actual focal spot size should be larger by a factor of $\sqrt{2}$. The pulse duration was measured by autocorrelation to be less than $30 \mathrm{fs}$. With these attributes the laser intensity at the focal point is on the order of $10^{14} \mathrm{~W} / \mathrm{cm}^{2}$, which is high enough to perform HHG in heavy noble gases.

\section{$4 \quad$ Harmonic generation with OPA}

We have performed HHG experiments with argon and xenon gases. Figure 4 shows the harmonic spectrum generated in xenon gas at different driving wavelengths but constant pulse energy of $50 \mu \mathrm{J}$. This figure clearly illustrates the tunability of the HHG signal. As shown in the figure, the frequency of the $37 \mathrm{th}$ harmonic of the $1.51 \mu \mathrm{m}$ fundamental is less than the frequency of the 35 th harmonic of the $1.22 \mu \mathrm{m}$ fundamental.

Assume the OPA output can be tuned between $\lambda_{1}$ and $\lambda_{2}$. If the $q$ th harmonic of $\lambda_{1}$ can be tuned to the adjacent harmonic of $\lambda_{2}$, i.e. $\lambda_{1} / q=\lambda_{2} /(q+2)$, the HHG spectrum above $\lambda_{1} / q$ will be fully tunable. Our OPA can be tuned between 1.1 to $1.6 \mu \mathrm{m}$. Therefore, we have demonstrated a completely tunable XUV radiation source from the 5 th harmonic $(0.22 \mu \mathrm{m})$ to the cutoff frequency. This is the first demonstration of full tunability in such a wide spectrum range with an OPA. Compared to the previously proposed tuning schemes, the wavemixing method requires precise temporal and spatial overlap of the strong fixed-wavelength pulses and the tunable weak OPA pulses $[14,15]$. The tuning scheme by non-adiabatic blueshift effect with intense short pulses ( $\sim 30 \mathrm{fs})$ can provide

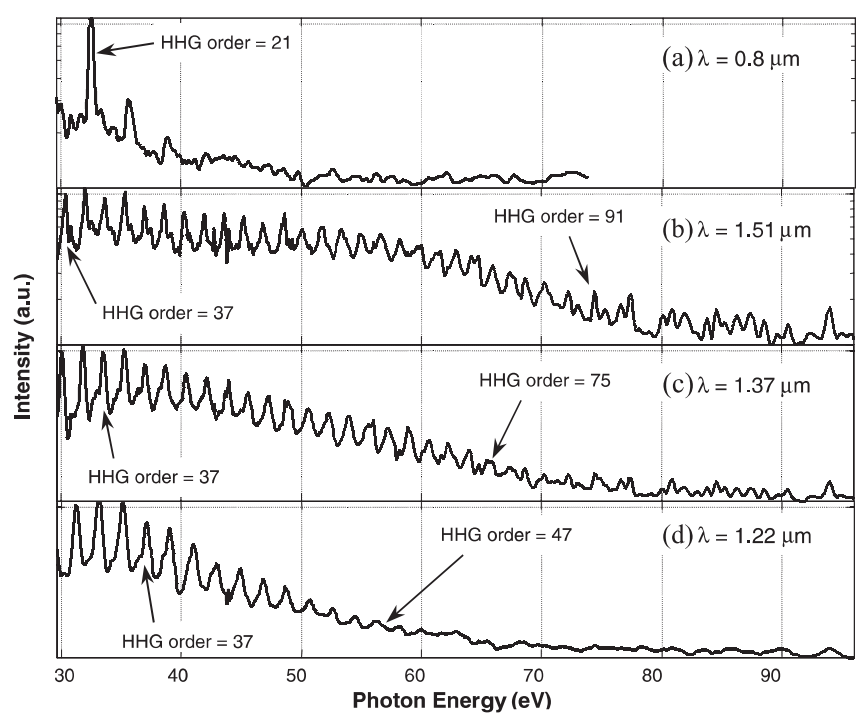

FIGURE 4 Harmonic spectrum using different driving field wavelengths, constant pulse energy $(50 \mu \mathrm{J})$ and focal spot size fully tunable HHG above $0.02 \mu \mathrm{m}[16,17]$. Our method here is more versatile and simple.

The results in Fig. 4 also demonstrated the cutoff dependence on the wavelength of the driving field. As shown in the figure, with the spectrums measured with $0.8,1.22,1.37$ and $1.51 \mu \mathrm{m}$ driving field, the longer driving wavelength always results in a higher cutoff photon energy. We also measured the harmonic spectrum generated in argon gas by $0.8 \mu \mathrm{m}$ and $1.51 \mu \mathrm{m}$ driving laser pulses. The pulse energy in both cases was $100 \mu \mathrm{J}$. The cutoff photon energy with $0.8 \mu \mathrm{m}$ driving field was $64 \mathrm{eV}$, while the cutoff using the $1.51 \mu \mathrm{m}$ pump field was $160 \mathrm{eV}$. Therefore, at constant driving intensity, the cutoff photon energy using a long-wavelength pump field is much higher than that found using $0.8 \mu \mathrm{m}$ from a Ti:sapphire laser.

For a given atom, the highest useful intensity is set by the saturation of ionization of the atom. The saturation ionization intensity is wavelength-independent in the tunneling ionization regime, therefore, for a given atom, the highest cutoff energy is strongly depend upon the driving field wavelength. This is shown explicitly in the following formula [10]:

$$
h v_{\text {cutoff }}=I_{\mathrm{p}}+\frac{0.5 I_{\mathrm{p}}^{3+a} \lambda^{2}}{\left[\ln \frac{0.86 \Delta t 3^{2 n^{*}-1} G_{l m} C_{n^{*} l^{*}}^{2} I_{\mathrm{p}}}{-\ln (1-p)}\right]^{2}}
$$

where $I_{\mathrm{p}}$ is the ionization potential and $\Delta t$ and $\lambda$ are the duration and the wavelength of the laser pulse, respectively. $l$ and $m$ are the orbital and magnetic quantum number. $n^{*}$ is the effective principal quantum number, $l^{*}=l-n^{*} \cdot p=98 \%$ is the ionization probability at the peak of the pulse. $a=0.5$ is a correction of the analytical approximation.

According to this equation, $\sim 2 \mathrm{keV} \mathrm{HHG}$ can be obtained by using a $1.6 \mu \mathrm{m}, 25$ fs driving field with helium gas, which is much higher than the highest harmonics $(0.5 \mathrm{keV})$ generated so far by a $0.8 \mu \mathrm{m}$ laser. Work is in progress to increase the OPA intensity so that the expected keV HHG emission can be generated by driving helium gas with an OPA at $10^{15} \mathrm{~W} / \mathrm{cm}^{2}$.

\section{$5 \quad$ Conclusions}

We have reported high harmonic generation using the output of an OPA. This is the first demonstration of a fully widely tunable XUV/soft X-ray HHG signal. The experimental system was constructed with a $\mathrm{kHz}$ gas valve and differential pumping vacuum chambers. This allowed a high vacuum in the detector chamber and a more efficient HHG experiment. Driven by the output of the OPA, the HHG experiments were performed with xenon and argon gases. The results show that the HHG photon energy was fully tunable from $0.22 \mu \mathrm{m}$ to the cutoff energy. We also confirmed that the HHG cutoff energy is significantly increased by increasing the driving field wavelength, as predicted by the cutoff law. For argon gas, the cutoff energy was increased from $64 \mathrm{eV}$ to $\sim 160 \mathrm{eV}$ when a $1.51 \mu \mathrm{m}$ driving field was used instead of a $0.8 \mu \mathrm{m}$ driving field from a Ti:sapphire laser. We expect that with a higher-power OPA, we will be able to produce $\mathrm{keV}$ coherent radiation through HHG in helium gas.

ACKNOWLEDGEMENTS This work is supported by the National Science Foundation through the STC Center for Ultrafast Optical 
Science. It is also supported by the Office of Basic Energy Science, Division of Chemical Science, Department of Energy under Grant No. DE-FG0200ER15082. We acknowledge Dr.P. Heimann, Lawrence Berkeley National Laboratory, for loaning us the X-ray spectrometer. We thank L. Walker, Clark MXR, for helping with the OPA.

\section{REFERENCES}

1 P. Salieres, A. L'Huillier, P. Antoine, M. Lewenstein: Adv. At. Mol. Opt. Phys. 41, 83 (1999)

2 T. Brabec, F. Krausz: Rev. Mod. Phys. 72, 545 (2000)

3 A. McPherson, G. Gibson, H. Jara, U. Johann, T.S. Luk, I. McIntyre, K. Boyer, C.K. Rhodes: J. Opt. Soc. Am. B 4, 595 (1987)

4 M. Ferray, A. L'Huillier, X.F. Li, L.A. Lompre, G. Mainfray, C. Manus: J. Phys. B 21, L31 (1988)

5 M. Bellini: Appl. Phys. B 70, 773 (2000)

6 P.B. Corkum: Phys. Rev. Lett. 71, 1999 (1993)

7 K.C. Kulander, K.J. Schafer, J.L. Krause: In: Super-Intense Laser-Atom Physics, Vol. 316 of NATO Advanced Study Institute, Series B: Physics, ed. by B. Piraux, A. L'Huillier, K. Rzazewski (Plenum, New York 1993) p. 316
8 O. Albert, H. Wang, D. Liu, Z. Chang, G. Mourou: Opt. Lett. 25, 1125 (2000)

9 M. Schurer, Z. Cheng, M. Hentschel, G. Tempea, P. Kalman, T. Brabec, F. Krausz: Phys. Rev. Lett. 83, 722 (1999)

10 Z. Chang, A. Rundquist, H. Wang, M.M. Murnane, H.C. Kapteyn: Phys. Rev. Lett. 82, 2006 (1999)

11 C. De Lisio, C. Altucci, R. Bruzzese, S. Solimeno, M. Bellini, P. Foggi: Appl. Phys. B 64, 323 (1997)

12 A. Flettner, J. Günther, M.B. Mason, U. Weichmann, R. Düren, G. Gerber: Appl. Phys. B 73, 129 (2001)

13 L.A. Lompre, M. Ferray, A. L'Huillier, X.F. Li, G. Mainfray: J. Appl. Phys. 63, 1791 (1988)

14 M.B. Gaarde, P. Antoine, A. Persson, B. Carre, A. L'Huillier, C.G. Wahlstrom: J. Phys. B 29, L163 (1996)

15 H. Eichmann, S. Meyer, K. Riepl, C. Momma, B. Wellegehausen: Phys. Rev. A 50, R2834 (1994)

16 C. Altucci, R. Bruzzese, C. De Lisio, M. Nisoli, S. Stagira, S. De Silvestri, O. Svelto, A. Boscolo, P. Ceccherini, L. Poletto, G. Tondello, P. Villoresi: Phys. Rev. A 61, 21801 (1999)

17 H.J. Shin, D.G. Lee, Y.H. Cha, J.-H. Kim, K.H. Hong, C.H. Nam: Phys. Rev. A 63, 53407 (2000) 